Peter Gärdenfors

JANA HolsanOva

\title{
COMMUNICATION, COGNITION, AND TECHNOLOGY
}

\section{Introduction}

The most likely form taken by the first human communication was gestures and miming. The body is still an important component in a direct conversation with other people. However, humans are the only animals who have developed a spoken language as our primary tool for communication. Dialogue is our most genuine form of interaction and it will be our point of departure when we compare different forms of communication supported by technology.

Since early in the history of Homo sapiens, we have used different media for communication. The oldest are cave paintings that are about 40000 years old. But it is above all written language that has influenced our way of mediating thoughts. The oldest forms of writing are about 5000 years old.

The last few centuries have seen rapid development of different technologies for communication. Printing was invented a little more than 500 years ago. Telegraphy and photography are about 150 years old. Bell invented the telephone in 1876, Marconi made the first radio transmissions in 1895, Edison taught us how to record sound 100 years ago, and moving pictures are equally old. In the last 50 years we have seen how the fax, the television, the computer and the mobile phone have radically influenced our ways of communicating with other people.

Imagine, for example that your boyfriend or girlfriend travels to New York to study for a semester. Unfortunately, you are unable to go along. What types of communication technologies will you use to keep your love alive? A handwritten letter is of course very personal, but it will take time before it reaches the addressee. E-mail is an excellent form of communication over long distances for keeping in touch with people; it is fast, cheap and you can write long messages. 
Texting an SMS by mobile phone is not a bad solution but is more suitable for short messages. However, if you want to express your feelings and get an immediate response, the telephone (or the videophone) is the superior medium to "feel close to" the other person. A disadvantage is of course the time difference: you cannot call at any time that suits you.

However, the different technologies will influence how the messages are shaped. In most respects, mediated communication is more limited than a normal dialogue, but as we shall see, there is an increasing number of methods to augment human communication with the aid of technology.

We will use face-to-face dialogue as a starting point when we analyse and compare different ways of using technology for communication. In section 2 , we will formulate a number of criteria that are useful for the analysis. In section 3, we will then present some of the most common types of technology-supported communication.

In a dialogue, the participants construct a common ground that will form the basis for how the conversation develops (Clark, Brennan, 1991). The common ground consists of background knowledge as well as the physical environment and the information generated in the course of the dialogue. Even the expectations that the speaker and the listener have of each other's thoughts, what is called theory of mind, belongs to the common ground. In technology-supported communication, where the participants are not located at the same place or are not communicating at the same time, there is no surrounding environment to build on and therefore the common ground will be more limited.

The speaker takes the common ground as given when she chooses what to say and how to say it. The listener assumes that the message is relevant in the sense that it conveys something new in relation to her previous knowledge and that what is communicated is important for the receiver (Grice, 1975; Sperber, Wilson, 1995).

\section{Criteria for analysing communication}

\subsection{Codes}

When comparing different forms of communication it is useful to distinguish between the contents of the information (what it means), which code it is expressed in, and which medium is used in the transmission (see e.g. Glass,

\section{0 \& COgNItIVE SCIENCE}


Holyoak, 1986: 8-10). The first factor that we shall consider in our analysis of communication concerns the choice of code.

The dominant code in human communication is of course language. There are a (rapidly decreasing) number of natural languages in the world that are used for spoken communication and somewhat fewer that have a corresponding written language. In addition, there are some artificial languages such as Esperanto, Ido, and Klingon, although they have not had any real impact. The grammars and vocabularies of the different languages vary considerably and are therefore different codes. However, there are no substantial differences in what can be expressed by the languages.

But a dialogue does not consist only of words. Gestures and miming are other "codes" that complement spoken language. It is more difficult to identify the different "signs" that are used in gesturing than to identify the words used in language. But for facial expressions there are fairly clear signals such as raising your eyebrows to signal surprise, and a wrinkle between your eyebrows means disliking or not understanding.

An important part of a dialogue is the eye contact that is established between the participants. This is essential for turn-taking and feedback in discourse. By eye contact the participants regulate whose turn it is to speak, confirm that they have mutual attention and interest.

Another important part of a conversation is the prosody - intonation, rhythm, and emphasis. We can express various emotional states, but also mark what kind of speech act is performed (statement, question, imperative, plea, announcement, etc.). In a conversation, a song might be an odd form of communication, but music is obviously also a code.

A conversation where the participants are physically present is multimodal: the participants point spontaneously to the objects in the environment, they nod, draw, gesticulate and touch each other. This functions because they have a joint perceptual space as an essential part of the common ground (Allwood, 2002; Holmqvist, Holsanova, 2007). The participants may also use other kinds of visual codes than those that belong to body language. For example, they can draw a road description as a clarifying complement to their oral instructions, present a photo of their children when talking about them or point to the timetable when discussing travel plans. Within modern forms of technology-supported communication, pictures and other visual tools play a more important role than they do in ordinary conversations. We will return to this below. 


\subsection{Medium}

The medium of a form of communication is the physical substrate that carries the message. However, "media" is used in a broader sense to refer to the technical systems that "mediate" the communication: radio, television, film, video and newspaper are all called media. As regards spoken dialogue, it is literally the sound waves in the air that are the medium. Later, various technical achievements have conveyed the sound vicariously via telephone or computer wires or via radio waves. The sound of a conversation fades away quickly, but it can be stored on different media: tape, $\mathrm{CD}$, hard disk, electronic memory in answering machines, etc.

Gestures and facial expressions are mediated by light to the eyes and they disappear immediately if they are not stored with the aid of some form of camera. Even eye contact is visual (in the blind it is replaced by tactile contact). Written language is also visual and can be stored and transmitted via paper, photos, or computers as media. The same also applies, of course, to various kinds of pictures, graphs, and diagrams. The tactile sense modality is sometimes used in dialogues, since touching may be part of the body language. The deaf-blind use a sign language that is shaped in the hand of the receiver so that he or she can feel the signs.

\subsection{Space}

The most important difference between dialogue and mediated dialogue is that the participants need not see each other or be within hearing distance. For modern technology, the distance between the communicators is of marginal significance: The communicators may as well be in two neighbouring rooms as on opposite sides of the planet.

In a virtual world, for example Second Life, a special form of spatial community can be achieved. In this world, the participants communicate via avatars that are present at the same place in the virtual world. In this form of dialogue, the participants can refer to what is present in the virtual environment (which makes the dialogue closer to natural) but they cannot refer to the real spaces they are located at.

\section{2 \&) COGNITIVE SCIENCE}




\subsection{Time}

An ordinary dialogue is immediate in the sense that the message reaches the listener directly and the responses of the listener, in the form of eye contact, facial expressions and humming, can have an immediate effect on what the speaker says. Normally, we are not aware of how synchronized we are during a conversation, but a telephone connection where the sound is delayed half a second is sufficient to cause serious disturbances of the dialogue.

In contrast to natural dialogue, the messages are delayed in many technology-supported systems. A letter may be delivered to the addressee weeks after it has been sent and the reply may take an equally long time. Modern communication systems are faster, but the delay still considerably influences the form of the messages. It makes a big difference to talk to somebody on the phone and to talk to each other via answering machines.

An important difference between immediate and delayed communication is that in a direct dialogue, the speaker cannot spend much time in selecting the right wording, while there is time to think through how you want to express yourself in a delayed exchange. It is even possible to change one's mind and rephrase the message before it is sent off. As we shall see in section 3.2, there are many differences between written language and speech, partly as a consequence of the delay in space and time. In other written forms of communication, where the delay is not equally long, such as chat, SMS or e-mail, the linguistic forms are closer to those of spoken language.

\subsection{Audience}

Even if direct dialogue is the most genuine form of communication, spoken language has, of course, always been used to address large audiences. Also when you speak to many people, you receive feedback from the audience, but there are differences in comparison to what happens in a dialogue: For example, the speaker can not have eye contact with everybody in the audience and it is not possible to build on such a rich common ground as in a dialogue. It is difficult for single listeners to signal that they do not understand or that they want to know more. The larger the audience, the less the overlap between the speaker 
and the listeners' inner worlds and consequently the less the common ground. Therefore, the expressions used by the speaker must be clearer and less ambiguous so that he can make sure that the listeners follow. If the audience consists of unknown persons, it becomes even more difficult for the speaker to be relevant and to construct a common ground.

The same factors govern mediated communication with one or many. It you write a letter, you can build on the common ground you have with the addressee and hence much can be left implicit. If you write a newspaper article, you can only presume what is commonly known at the time of writing. If you write a book that you expect to be read over a period of many years, you cannot build on dated information. Consequently, more must be stated explicitly in the text. Similar differences apply to telephone conversations in comparison to radio broadcasting.

\subsection{Interactivity}

An ordinary dialogue is interactive in the sense that one partner can immediately react to the other. The interactivity of a mode of communication can be defined as the possibilities for the receiver to influence the contents (or the form) of the continued communication (Jensen, 1998: 232). A traditional radio broadcast is an example of a mode of communication with low interactivity, but if it is possible for the listeners to ask questions or request, say, a piece of music, interactivity is already improved.

The time delay in turn-taking is also a factor that influences the degree of interactivity. Three types of written exchanges can be compared: An exchange of letters is less interactive than an exchange of e-mails, which in turn is less interactive than chat. Yet another factor that influences the degree of interactivity is the mobility of the communication system. A mobile phone provides better interactivity than an ordinary telephone since the people who communicate become less dependent on where they are physically located. In the same way, a laptop provides better interactivity than a stationary computer in relation to e-mail and chat. A communicative disadvantage of portable platforms is that you do not know where your dialogue partner is located. The lack of spatial information in mobile phones results in a more restricted common ground of the communicators. This leads to frequent questions of the type "Where are you?" among users of mobile phone, which are not relevant at all when ordinary phones are used. In

\section{4 \&) COGNITIVE SCIENCE}


general, however, the increased interactivity of a particular mode of communication compensates for the shortcomings that are created by the displacement in space and time.

\section{Properties of different kinds of communication}

We will now use the criteria presented in the previous section to analyse various types of communication. The rich availability of technical possibilities that exist today makes it possible to choose different kinds of communication media for different types of messages. For example, we choose a medium depending on how quickly we must communicate. E-mail and in particular letters are not used in urgent situations, but then mobile phone conversation or SMS is used since the receiver is supposed to be reached immediately. If not even this works, you yourself or a messenger must physically move to the receiver. Another example is that there is a tendency to send an SMS rather than calling late at night. This is primarily because you will not disturb the receiver (who may be asleep) to the same extent as if you had made a regular call.

\subsection{Direct conversation}

Dialogue, face to face, is fundamental for human communication. It is important to note, however, that a dialogue is most of the time not a purpose in itself, but it is used in connection with some other activity that the participants are involved in: when they are solving a problem, arguing to reach a joint decision, giving each other instructions, etc. A dialogue is a part of a common "project" (Clark, 1996) - planning a party, shopping together or deliberating on which movie to watch.

A dialogue is an interactive process of shared control where the participants must coordinate their linguistic and non-linguistic actions while they are performing various common activities. On the one hand, coordination is about how the dialogue should be organised by turn-taking. On the other hand, coordination deals with how various topics are introduced in the discussion and how they are concluded. When speakers make a mistake or perceive that something is not clear, they interrupt themselves and correct what has been said. Such repairs may 
concern pronunciation, the choice of words or grammar. Dialogue is a tool for achieving something common: The participants negotiate what steps to take, what aspects to discuss in order to solve a problem, what criteria to choose to judge the alternatives and to evaluate the solution (Wästerfors, Holsanova, 2005).

A dialogue is a dynamic process where the participants catch the thoughts of the other and meet and expand them in their own contributions. The focus of consciousness is continuously moved, primarily by language steering the attention of the participants, either towards factors in the environment or towards something in the common "inner world" (Chafe, 1994). The partners successively construct their understanding: statements are confirmed, legitimated, challenged, developed, corrected, declared invalid, etc. (Linell, 2005). Feedback plays a central role for signalling understanding, agreement, encouragement, and continued interest (Allwood et al., 1992).

A dialogue can be seen as a form of distributed cognition (Hutchins, 1995; Linell, 2005). Each individual does not have to consider everything by himself or herself since the conversation partners help each other to find words or phrases. In the words of Linell (1998: 224): "In a dialogic situation part of the thought has already been thought by the other and the speaker may exploit this". Because several persons are working on the same problem, the participants can automatically access parallel cognitive processes in the form of increased attention, improved memory and deeper processing of information. Not only is the responsibility for remembering the different steps, solutions and topics distributed between the participants, but they also take turns in taking initiative and in giving feedback. Another advantage is that misunderstandings can be repaired interactively and unclear statements can be corrected immediately, which leads to a quicker development of the dialogue.

\subsection{Letters and other uses of written language}

In early human history, messengers were used to communicate over long distances. The speaker sent another person who conveyed the message to the receiver. Written language made the personal messenger superfluous. A letter is a visual form of communication that is permanent and enables comparatively cheap communication over long distances. A limitation is that feedback is slow. The messages may be long and the text is often well planned. A letter can be read by others and it thereby has a more public character than an ordinary conversation. It is difficult to whisper in a letter.

\section{6 \&) COGNITIVE SCIENCE}


Written language has limitations, however. A dialogue is bound to a certain context and is expressed with the aid of prosody and facial expressions that may be more intense than the "literal" message. All this is lost when language is fixed on a sheet of paper. It took some time before written language developed into an independent form of communication - it was long seen merely as a support for what was to be said. During the Middle Ages, reading was equal to reading aloud - the text was transformed into the oral (McLuhan, 1964: 83). Only after the invention of printing did silent reading develop.

Written language must compensate, by various means, for the parts of the communication that are transmitted by prosody, rhythm, and emphasis. Olson (1994) shows that linguistic expression of speech acts, such as "submit", "explain”, and "suggest”, also arose during the Middle Ages. These markers are not needed in an oral tradition where the speech act is expressed directly using prosody and other tools. Another example is that it is more difficult to express irony in written language than in speech.

A consequence of the permanence of writing and the liberty of taking one's time when formulating a text is that the sentence structures in written communication become more advanced, with more difficult words and heavy syntactic constructions (Linell, 1978). The reader has plenty of time to interpret the text and can in the worst case use a dictionary.

When the telegraph was invented, news could reach the audience very fast, which made the whole world more present. It is interesting to note that the form of telegraphic messages in turn influenced the language in newspapers which, having been more like letters, became briefer and more proclaiming. Fax has most of the properties in common with letters, except that it is transmitted faster than ordinary mail. This form of communication also tends to be less private - you would hardly send a love letter via fax.

\subsection{SMS, e-mail, and chat}

SMS is a visual medium that supports urgent communication. It is characterized by short planning time and is suitable for quick, short, contact-creating or co-ordinating messages. SMS does not require the simultaneous presence of the receiver and is relatively permanent, depending on how much is saved. Among the disadvantages are that the messages contain a limited number of symbols and that the method of input is cumbersome and time-consuming. 
E-mail is a visual medium that allows long messages and is therefore used for informative purposes. New e-mail systems and MMS amplify written communication by the possibility to send pictures, graphic information, and sound files. The writer uses a comparatively long time to formulate the message before it is sent, and the partners can reply with a delay. The message is often directed to one or more persons you know and who constitute a homogeneous group with a rich common ground. The language of e-mails is similar to that of ordinary letters. However, the method of writing and the survey of the text is better than in SMS.

Instant messaging (chat) is characterized by speed and spontaneity and supports written communication in real time with immediate feedback. This makes it come close to dialogue, but in contrast to the fleeting dialogue, instant messaging has more permanence. The messages remain on the screen during the conversation, but are in general deleted when the communication window is closed. It is therefore possible to directly refer to earlier messages - at least as long as the chat continues.

In SMS, e-mail and chat, written language is adapted in order to communicate emotional qualities that are found in a dialogue. Computer-supported language is not just something in between text and speech, but is in many respects very similar to speech (Ko, 1996). The writers feel the pressure to write fast (preferably as fast as they speak) and do not have time to plan or reflect. They compensate for voice quality, facial expressions, and gestures by using smileys (e.g. ;o), asterisks for actions (*blinks*, *smiles*), unconventional punctuation (...!?), abbreviations (4U) and capitals for emphasis (SHE gave ME a gift). As a consequence of the rapid turn-taking, the sentences have less linguistic variation and a simpler construction. On the other hand, the users are tolerant and do not care whether the language has the same degree of perfection as in (classical) written language. Politeness is also less of a constraint. A user can, for example, stop a chat very abruptly without being seen as impolite.

\subsection{Telephone, mobile phone, and voice messages}

The telephone offers an acoustic and fleeting medium that affords simultaneous personal communication over a distance. The medium is suitable for contact-seeking, informative, emotional, and persuasive communication, with direct feedback. The receiver can hear what the speaker says and how it is said, but does not see the facial expressions, the gestures, and the body language. In long-dis-

\section{8 \&, COGNITIVE SCIENCE}


tance calls there is sometime a time lag that immediately disturbs the feedback and the turn-taking in the conversation.

The mobile phone is a portable tool for communication that drastically increases the possibility of reaching a communication partner at the time you desire - as long as the partner has the phone turned on. As never before, it is now possible for us to have direct dialogues with almost everybody at almost any time. Mobile phones also offer time-independent messages via the voice mail and written communication via SMS or e-mail.

In contrast to the telephone, the voice mailbox is a permanent medium without interactivity or immediate feedback. These properties explain why it is not suitable to break up from your boyfriend via a voice mail: the permanence means that the receiver can replay the message several times; the lack of common context often leads to problems in finding the correct interpretation of the message; and the lack of interactivity does not give the dumped person any answers to his questions.

\subsection{Videophone and video-conference}

Videophone (e.g. Skype) and video-conferences are examples of advanced technology-supported communication with a high degree of interactivity. They allow the use of language, gestures, and body language and thereby provide dialogue-like conversations over long distances. The technology creates the impression that the communication partners share the same room and they may use a complete register of verbal and non-verbal signals in their normal functions. Hand in hand with this come also our expectations of video conversations. Eye contact is an essential part of the non-verbal communication. The communicator who uses a system with video and sound link may believe that the same rules apply as in communication face to face, for example that communication can be initiated and attention drawn via eye contact. However, eye contact does not function in the same way in a video conversation because of the camera placement. Current technology does not allow that you attend to the screen and at the same time look into the camera. If you try to establish eye contact via the screen, your communication partner will see your eyes staring to the floor or out in the air.

If the implicit rules are not followed, irritation will soon follow. Therefore, the users instead try to "stare out" the other one, wave, exaggerate movements, or 
grimace. The consequence is that technology does not support what it purports to and the users are disappointed (Hutchby, 2001; Heath, Luff, 1991). If, in addition, the bandwidth and computer power is too low to display real-time video in high resolution, turn-taking will not work well, faces will be distorted, body language cannot be perceived and certain gestures can be missed.

In spite of the rapid development of communication technology, we still travel far for various kinds of meetings. For example, when it concerns important business negotiations or marriage proposals, we still want to have direct contact with our dialogue partner. The direct conversation offers certain properties that technology cannot yet replace. Some researchers argue that the smell of the other is an underestimated factor in a dialogue.

\subsection{Augmented communication}

Already when we use a paper for drawing on as a complement to an oral road description, we use "augmented" communication (Diderichsen, 2006), in the sense that we add a medium that is not necessary for spoken language. Someone who uses a dictionary to understand a letter also augments the communication.

The steadily increasing access to the Internet has provided us with a powerful tool for further forms of augmentation. If, for example, both participants in a video conversation simultaneously are connected to the Internet, they can during the conversation gather facts, check what the partner claims, and supplement the discussion with pictures and other non-verbal information. It becomes more difficult to be relevant in an augmented communication situation.

Within computer-supported collaborative work various programs are used so that persons located at different places can work simultaneously with the same material. For example, two architects can have the same drawing on their screens and communicate via words or point or draw. Nowadays, one finds new techniques based on communicators sharing various interactive displays. There are systems where a projector in the ceiling displays pictures of documents on a table. The system can also detect how hands move over the table and in this way the persons around the table can "pull", "open" and "close", and in other ways interact with the virtual objects that are projected.

In the future we will meet more kinds of augmented communication. There may be virtual food and drink on the restaurant table where you can order by

\section{0 \&) COgNitive SCIENCE}


"pulling" the food or drink to your plate and find out about the ingredients by double clicking on it. The interactive environments of Blade Runner and other science fiction movies are getting closer.

\subsection{Communication with handicap}

Technology-supported communication has led to remarkable changes for people with different handicaps. A spellchecking program suffices to make it possible for a dyslectic to become more secure in his or her written communication. Advanced technology aids are available for people with aphasia (Kitzing et al., 2005) and computer-supported communication programs with pictures and symbols facilitate communication for disabled or speech-handicapped persons in general (Rydeman, Zachrisson, 2001). Braille, and its corresponding technology, has made it possible for blind people to replace the visual paper-based communication by a tactile medium. The telephone must have been a revolution for the blind and the mobile phone makes communication over distance even easier for them. During the last few decades speech synthesis programs that can read e-mail, web pages and newspapers have to some extent replaced the function of Braille. For sure, the synthetic voice is still a bit robotic, but it is becoming more and more human.

Deaf persons can of course use letters and text-based media to communicate over a distance. Using the telephone for a long time involved using a messenger. During a period, the text telephone has been a tool for the deaf - it can be seen as an early chat function. Computers with e-mail and chat are of course an improvement but dependent on having a computer available. A paradoxical consequence of the development of mobile phones is that is has radically facilitated the communication of the deaf. SMS allows a rather quick and interactive communication, but above all the videophone has made it possible for the deaf to communicate in real time over long distances via sign language.

\section{References}

Allwood, J. (2002). Bodily communication dimensions of expression and content. In: B. Granström et al. (eds.), Multimodality in Language and Speech Systems (pp. 7-26). Dordrecht: Kluwer Academic Publishers.

Allwood, J., Nivre, J., Ahlsén, E. (1992). On the semantics and pragmatics of linguistic feedback. Journal of Semantics 9(1): 1-26. 
Chafe, W. (1994). Discourse, Consciousness, and Time: The Flow of Displacement of Conscious Experience in Speaking and Writing. Chicago: The University of Chicago Press. Clark, H.H. (1996). Using language. Cambridge: Cambridge University Press.

Clark, H.H., Brennan, S.E. (1991). Grounding in communication. In: L.B. Resnick, J.M. Levine, S.D. Teasley (eds.), Perspectives on Socially Shared Cognition (pp. 127149). Washington, DC: American Psychological Association.

Diderichsen, P. (2006). Augmented communication: The communicative potential of the internet. Lund: Lund University Cognitive Studies 132.

Glass, A.L., Holyoak, K.J. (1986). Cognition. $2^{\text {nd }}$ edition. New York: Random House.

Grice, P. (1975). Logic and conversation. In: P. Cole, J.L. Morgan (eds.), Syntax and Semantics, vol. 3: Speech Acts (pp. 41-58). New York, NY: Academic Press.

Heath, C., Luff, P. (1991). Disembodied conduct: Communication through video in a multi-media office environment. Proceedings of the SIGCHI conference on human factors in computing systems: Reaching through technology (pp. 99-103). New Orleans, LA.

Holmqvist, K., Holsanova, J. (2007). Embodied communication and gestural contrast. In: E. Ahlsén et al. (eds.), Communication - Action - Meaning. Göteborg: Department of Linguistics, Göteborg University.

Hutchby, I. (2001). Conversation and Technology: From the Telephone to the Internet. Cambridge: Polity.

Hutchins, E. (1995). Cognition in the Wild. Cambridge, MA: MIT Press.

Jensen, J.F. (1998). Interaktivitet og interaktive medier. In: J.F. Jensen (ed.), Multimedier, hypermedier, interaktive medier, vol. 3 of FISK serien (pp. 199-238). Aalborg: Aalborg Universitetsforlag.

Kitzing, P., Ahlsén, E., Jönsson, B. (2005). Communication aids for people with aphasia. Logopedics Phoniatrics Vocology 30: 41-46.

Ko, K.-K. (1996). Structural characteristics of computer-mediated language: A comparative analysis of inter change discourse. In: S. Herring (ed.) Computer-Mediated Discourse Analysis: The Electronic Journal of Communication 6 (3).

Linell, P. (1978). Talat och skrivet språk. In: P. Linell, Människans språk. En introduktion till språk, tänkande och kommunikation (pp. 230-235). Lund: Gleerups.

Linell, P. (1998). Approaching Dialogue: Talk, Interaction and Contexts in Dialogical Perspectives. Amsterdam: John Benjamins.

Linell, P. (2005). En dialogisk grammatik? In: J. Anward, B. Nordberg (eds.), Samtalets Grammatik (pp. 231-315). Lund: Studentlitteratur.

McLuhan, M. (1964). Understanding Media. New York, NY: McGraw-Hill.

Olson, D.R. (1994). The World on Paper. Cambridge: Cambridge University Press.

Rydeman, B., Zachrisson, G. (2001). Dynamiska kommunikationsprogram och styrsätt för personer med rörelsehinder. Projektrapport nr 2 - IT i Praktiken. Vällingby: Hjälpmedelsinstitutet.

Sperber, D., Wilson, D. (1995). Relevance: Communication and Cognition. $2^{\text {nd }}$ edition. Oxford: Blackwell.

Wästerfors, D., Holsanova, J. (2005). Examples as crucial rhetorical tools in discourse on 'others'. Text 25 (4): 519-554.

\section{2 \&) COgNItIVE SCIENCE}

